\title{
Understanding the routes of metastasis in colorectal cancer
}

A newly developed mouse model of colorectal cancer (CRC) has demonstrated that liver metastases can develop without prior lymph-node involvement. This finding could have important therapeutic implications regarding the staging of this disease.

Many patients with CRC are actually more likely to die as a result of metastases in distant organs (for example, the liver or lungs) than their primary tumour. However, understanding the mechanisms and routes of metastasis in CRC has been difficult, particularly given the lack of a suitable model of metastatic CRC. There has been some confusion as to whether liver metastases can develop without previous lymph-node involvement.

Kevin Leong and colleagues investigated this issue by developing a novel mouse model of spontaneous metastatic lesion formation. The researchers had several criteria in mind that the model should fulfil: first, the primary tumour must be localized in the colon; second, the primary tumour must originate from the mucosal layer of the colon wall (as in human CRC); third, single intact tumours should be implantable on the mucosal layer (to preserve interactions of tumour cells with stromal cells and the extracellular matrix); and fourth, metastatic lesions should be detectable in the liver, lungs and lymph nodes (again, mimicking human CRC).

To satisfy these criteria, the team developed a novel lumen implantation technique involving induction of rectal prolapse followed by implantation of a single donor tumour fragment on the mucosal surface. This model was then able to demonstrate that cancer cells at the colon were able to spread directly to the liver without prior lymphatic spread.

"Our findings have important implications for clinical disease staging, as lymph-node-positive stage III and distant-metastasis-positive stage IV CRCs may be regarded as parallel progressions from localized stage II disease, rather than sequential progressions as they are commonly viewed today," explains

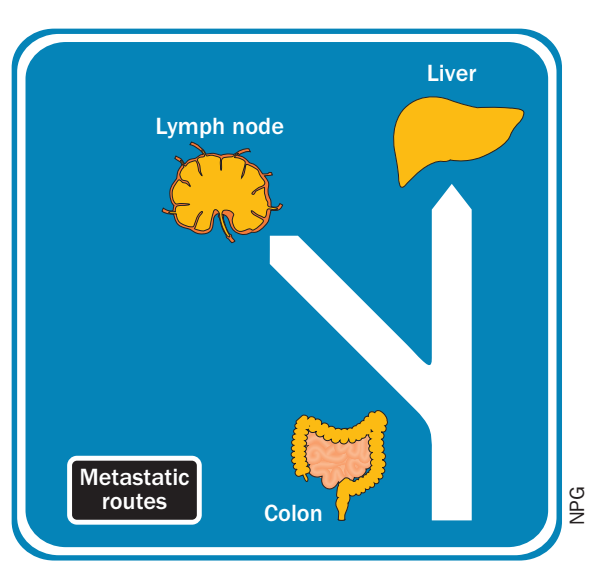

Leong. The researchers also hope that their implant model might aid drug development, particularly therapies aimed at targeting metastasis. Indeed, they are already using their model for pre-clinical testing of candidate therapeutics.

Isobel Leake

Original article Enquist, I. B. et al. Lymph nodeindependent liver metastasis in a model of metastatic colorectal cancer. Nat. Commun. 5, 3530 (2014) 